\title{
Risk Stratification of Locally Advanced Non-Small Cell Lung Cancer (NSCLC) Patients Treated with Chemo-Radiotherapy: An Institutional Analysis
}

This article was published in the following Dove Press journal: Cancer Management and Research

\author{
Amna Sher' \\ Sowmini Medavaram' \\ Barbara Nemesure ${ }^{2}$ \\ Sean Clouston ${ }^{2}$ \\ Roger Keresztes'
}

'Department of Medicine, Stony Brook University Hospital, Stony Brook, NY, USA; ${ }^{2}$ Department of Family, Population and Preventive Medicine, Stony Brook University Hospital, Stony Brook, NY, USA
Correspondence: Amna Sher Email Amna.Sher@Stonybrookmedicine. edu
Background: The purpose of this study was to determine which factors predicted survival and to derive a risk prediction model for patients with locally advanced non-small cell lung cancer (NSCLC) receiving concurrent chemo-radiotherapy (cCRT).

Methods: This investigation included 149 patients with locally advanced NSCLC who were treated with cCRT at Stony Brook University Hospital between 2007 and 2015. A finite set of demographic, clinical, and treatment variables were evaluated as independent prognostic factors. Kaplan-Meier survival curves were generated, and log rank tests were used to evaluate difference in survival between groups. To derive a risk score for mortality, a machine learning approach was utilized. To maximize statistical power while examining replicability, the sample was split into discovery $(n=99)$ and replication $(n=50)$ subsamples. Elastic-net regression was used to identify a linear prediction model. Youden's index was used to identify appropriate cutoffs. Cox proportional hazards regression was used to examine mortality risk; model concordance and hazards ratios were reported.

Results: One-quarter of the patients survived for three years after initiation of cCRT. Prognostic factors for survival in the discovery group included age, sex, smoking status, albumin, histology, largest tumor size, number of nodal stations, stage, induction therapy, and radiation dose. The derived model had good risk predictive accuracy $(\mathrm{C}=0.70)$. Median survival time was shorter in the high-risk group (0.93 years) vs the low-risk group (2.40 years). Similar findings were noted in the replication sample with strong model accuracy $(\mathrm{C}=0.69)$ and median survival time of 0.93 years and 2.03 years for the high- and low-risk groups, respectively.

Conclusion: This novel risk prediction model for overall survival in patients with stage III NSCLC highlights the importance of integrating patient, clinical, and treatment variables for accurately predicting outcomes. Clinicians can use this tool to make personalized treatment decisions for patients with locally advanced NSCLC treated with concurrent chemo-radiation.

Keywords: prediction model, survival, locally advanced, NSCLC

\section{Background}

Lung cancer is the most common cancer worldwide and remains the leading cause of cancer-related mortality, with a 5-year survival rate of $19 \%$. ${ }^{1}$ About $30-40 \%$ of patients with NSCLC have locally advanced disease at presentation. This is a heterogeneous population which includes potentially resectable tumors with microscopic metastases in lymph nodes to bulky unresectable tumors with multiple involved nodal stations. This makes it difficult to choose optimal treatment for individual patients. 
Radiation was the standard treatment for patients with locally advanced unresectable NSCLC for decades, but survival rates were less than $10 \%$. The Cancer and Leukemia Group B (CALGB) 8433 trial, in which sequential chemotherapy with cisplatin and vinblastine was followed by thoracic radiotherapy (TRT), showed improved survival over TRT alone. ${ }^{2}$ The median survival and 5-year survival rates were 9.7 months and $7 \%$ for TRT alone, compared with 13.8 months and $19 \%$ respectively in the sequential chemo-radiation arm $(P=0.006)$. RTOG 9410, a three-arm phase III trial, compared two concurrent cisplatin-based regimens and TRT to sequential chemotherapy and TRT. The five-year survival was significantly higher for the concurrent regimens when compared to sequential treatment. This established cisplatin based chemo-radiotherapy as the standard of care treatment for locally advanced NSCLC.

Subsequent trials have compared induction chemotherapy followed by cCRT with CRT alone, as well as consolidation chemotherapy after CRT. ${ }^{3-5}$ But none of the studies have shown any survival benefit with either approach.

In the early 2000s, Belani et al conducted a randomized trial incorporating carboplatin and paclitaxel with standard daily TRT in patients with locally advanced NSCLC to determine the optimal sequence of combined modality therapy. ${ }^{6}$ Concurrent weekly paclitaxel, carboplatin, and TRT followed by consolidation was associated with superior median OS of 16.3 months compared to the other two arms, at the expense of some increased toxicity. This regimen is relatively well tolerated and is commonly used in clinical practice.

Immunotherapy has revolutionized the treatment of lung cancer over the past several years, and antiprogrammed cell death protein 1/programmed cell death ligand 1 (PD-1/PDL-1) agents have emerged as novel treatments for NSCLC patients. The PACIFIC trial was the landmark trial in which consolidation immunotherapy with durvalumab, an anti-PDL-1 agent, in patients with locally advanced NSCLC following chemo-radiation was associated with significantly improved progression-free survival (PFS) and OS. ${ }^{7}$ Consolidation durvalumab is the major advancement in the treatment of stage III NSCLC patients in decades, and has become the new standard of care per current National Comprehensive Cancer Network (NCCN) guidelines.

Prior work has suggested that weight loss, tumor stage, performance status, and pulmonary function are pretreatment prognostic factors associated with survival in patients with NSCLC. ${ }^{89}$ Various studies evaluating the role of other variables such as age, gender, histology, tumor size, and radiation dose have shown inconsistent results. The objective of this study was to investigate prognostic factors for survival in NSCLC patients with locally advanced disease treated with CRT and to develop a risk stratification model based on these factors.

\section{Materials and Methods}

\section{Patient Population}

This investigation included a retrospective chart review of patients with unresectable locally advanced NSCLC, treated with chemo-radiotherapy at Stony Brook University from January 2007 to December 2015. This included medically inoperable stage II and stage III patients based on the American Joint Committee on Cancer (AJCC) 7th edition staging protocol, locally recurrent disease after initial surgery, and stage IV patients with disease limited to the thorax after resection of single distant metastasis. All patients were alive for at least one month after treatment initiation. In total, 151 patients met eligibility criteria. After excluding two who lacked survival analysis, the final sample included 149 unique patients.

Staging work up included complete blood count, basic metabolic panel, liver function tests, computed tomography (CT) of the chest, abdomen, and pelvis, positron emission tomography/computed tomography (PET/CT), bronchoscopy, or endobronchial ultrasound (EBUS). Magnetic resonance imaging (MRI) of the brain and Tc-99m whole body bone scans were performed depending on clinical indication and feasibility. Mediastinoscopy was not done routinely. Nodal stations were defined by PET-positive nodal stations. All the patients had pathologically confirmed disease. Resectability was determined by cardiothoracic surgeon and after evaluation at a multidisciplinary tumor board.

Patient records were reviewed to collect demographic data including age, gender, smoking history, Eastern Oncology Group (ECOG) performance status, histology, stage, number of nodal stations, chemotherapy regimen, and radiation dose ( $\leq 54 \mathrm{~Gy}, 54-60 \mathrm{~Gy}$ ).

\section{Radiation Treatment}

All patients underwent CT simulation for threedimensional radiotherapy planning. A total radiation dose of 60-66 Gy was planned for all patients. RT was delivered in 1.8 or 2 Gy fractions, five times a week. All 
patients received weekly platinum-doublet chemotherapy concurrent with radiation.

\section{Chemotherapy}

Most patients received carboplatin (AUC 2) and paclitaxel $\left(45-50 \mathrm{mg} / \mathrm{m}^{2}\right)$ weekly concurrently with radiation. One patient received docetaxel $\left(25 \mathrm{mg} / \mathrm{m}^{2}\right)$. Most patients received six weekly doses of chemotherapy (range 1 to 6). At our institution, consolidation chemotherapy was not routinely given and chemotherapy was delayed for hematological toxicity as per CTCAE criteria and based on the treating medical oncologist's clinical experience.

\section{Statistical Analysis}

Descriptive statistics are presented to characterize the study participants and include means, standard deviations (SD), and percentages (\%). Multivariable analyses relied on Cox proportional hazards models which were used to examine survival risk. The Efron method was used to handle ties, while Schoenfeld residuals were used to test the proportional hazards assumption; all analyses shown passed the proportional hazards assumption. Concordance (C) between model and outcome was utilized to examine model accuracy.

The goal of the risk score was to identify risk factors that predicted survival. To improve predictive reliability, the sample was therefore randomly divided into discovery and replication samples. Since the purpose of these two subsamples was different, power to detect significant effects also varied. Notably, since only the risk score was used in the replication cohort, power analyses were used to determine the optimal size for the replication cohort $(\mathrm{n}=50)$ with the remaining patients $(\mathrm{n}=99)$ serving as the discovery group.

In the discovery sample, the elastic net algorithm ( $\mathrm{n}$-folds $=40 ; n-\alpha / \mathrm{n}-\lambda=100$ ) was utilized to model the $\log$ survival time to identify a linear mixture of indicators that predicted survival and a multivariable risk score was calculated. ${ }^{10}$ The risk score was then used in the discovery cohort and Youden's method was used to stratify patients into high- and low-risk groups. ${ }^{11}$ In replication analyses, both the continuous and stratified risk score were used to predict survival. Hazard ratios, 95\% confidence intervals, and model concordance were reported for the risk score to show replicability with the discovery sample model. The stratified risk score was used to provide a Kaplan-Meier curve and to estimate sensitivity and specificity in the replication sample. All analyses were implemented utilizing Stata 15/SE (Stata Corp.).

\section{Results}

\section{Sample Description}

Table 1 depicts the characteristics of the study population $(n=149)$. The mean age was 65 years $(S D=10$ years $)$ with equal number of males and females, and the majority of the patients were smokers. The histology distribution indicated approximately $40 \%$ of patients with squamous cell carcinoma, $40 \%$ with adenocarcinoma, and $20 \%$ with otherwise unspecified histology. The mean ECOG performance status was $0.85(\mathrm{SD}=0.50)$ and most of the patients $(83.4 \%)$ had stage III disease. The mean albumin and hemoglobin values were $3.9 \mathrm{~g} / \mathrm{dl}$ and $12.3 \mathrm{~g} / \mathrm{dl}$, respectively. The average largest tumor size was $4.3 \mathrm{~cm}$, with the average of four involved

Table I Sample Characteristics of $N=149$ Lung Cancer Patients

\begin{tabular}{|l|l|l|}
\hline Continuous Variables & Mean & SD \\
\hline Age, years & 65.45 & 10.05 \\
Largest tumor size, cm & 4.30 & 2.32 \\
Albumin, g/dl & 3.92 & 0.47 \\
Hemoglobin, g/dl & 12.32 & 1.84 \\
Nodal stations, number & 3.97 & 2.78 \\
\hline Categorical variables & Percentage & \\
\hline Female sex & 51.01 & \\
\hline Performance status & & \\
0 & 20.27 & \\
I & 75.00 & \\
2+ & 4.73 & \\
\hline Current smoker & 97.32 & \\
Induction treatment & 15.44 & \\
Survived three years & 24.16 & \\
History of other cancer & 30.41 & \\
\hline Histology & & \\
Adenocarcinoma & 40.94 & \\
Squamous cell & 39.60 & \\
Not otherwise specified & 19.46 & \\
\hline Stage & & \\
II & 6.90 & \\
III & 83.45 & \\
IV & 9.66 & \\
\hline Dosage, Gy & & \\
S60 & 81.25 & \\
>660 & & \\
\hline
\end{tabular}

Abbreviations: SD, standard deviation; $\mathrm{cm}$, centimeter; $\mathrm{g} / \mathrm{dl}$, grams per deciliter; Gy, Gray units. 
nodal stations per patient. Most patients received a total radiation dose $\leq 60 \mathrm{~Gy}$. All patients received weekly platinum-doublet chemotherapy concurrent with radiation. Only one-quarter of the patients were alive at three years.

\section{Discovery Analyses}

Examining standardized effect sizes in the discovery sample (Table 2) identified a number of potential predictors including largest tumor size, albumin, hemoglobin, number of nodal stations, female sex, induction therapy, history of other cancer, stage, and radiation dose. Model fit analyses revealed that the saturated model was good at predicting overall survival compared with any specific measure $(C=0.71)$. Elastic-net analyses $(\alpha=0.10, \lambda=0.44)$ reduced this list to include age at treatment initiation, largest tumor size, albumin, number of nodal stations, sex, smoking status, histology, induction therapy, stage, and radiation dose (Table 2 includes estimated beta coefficients). The predicted risk score (mean $=0.319, \mathrm{SD}=0.352$ ) was able to predict survival $(\mathrm{HR}=8.56,[4.30-17.02]$, $P<0.001)$ and resulted in no loss of predictive power $(\mathrm{C}=0.70$; baseline survival $=0.693$, baseline hazard $=0.018)$. At the optimal determined cutoff (risk score $=0.65$ ), those in the high-risk group had much higher risk of mortality than those in the low-risk group ( $\mathrm{HR}=3.18,[1.85-5.44]$,

Table 2 Beta Coefficients Derived from the Elastic-Net Algorithm

\begin{tabular}{|l|l|}
\hline Patient Characteristics & Beta \\
\hline Age, years & 0.014 \\
Largest tumor size, cm & 0.097 \\
Albumin, g/dl & -0.54 I \\
Nodal stations, number & 0.127 \\
Female sex & -0.674 \\
Former smoker & -0.702 \\
Induction therapy & -1.05 I \\
\hline Former smoker & \\
\hline Histology & \\
Adenocarcinoma & \\
Squamous cell & 0.024 \\
Not otherwise specified & -0.517 \\
\hline Stage & \\
II & 0.583 \\
III & 0.000 \\
IV & 1.059 \\
\hline Dosage $>60$ Gy & -0.623 \\
\hline
\end{tabular}

Note: Beta coefficients are derived from a Cox proportional hazards specification with baseline survival of $0.34 \mathrm{I}$ and baseline hazards of 0.078 .

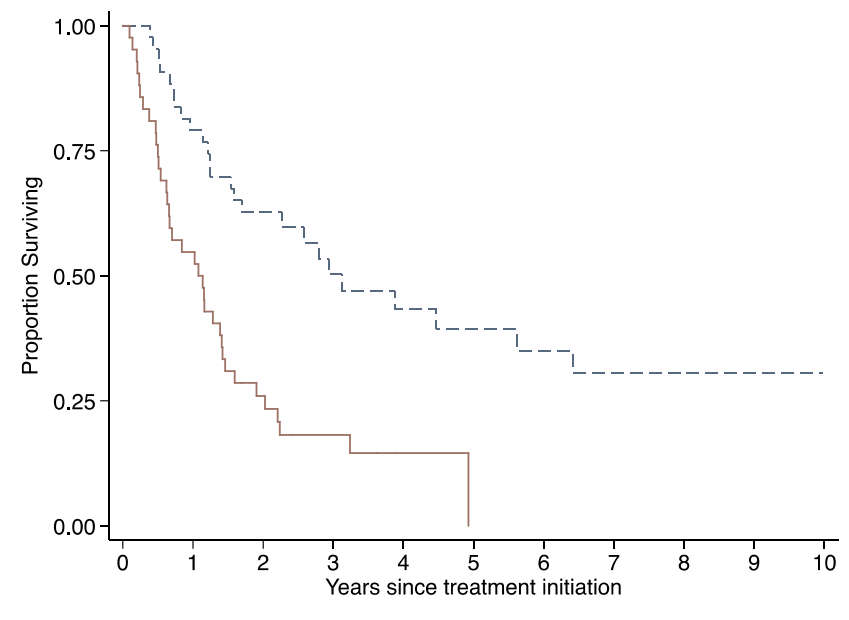

Figure I Kaplan-Meier analysis of survival risk in the high- and low-risk groups in the discovery sample. Dashed line shows survival for the low-risk group, while solid line shows survival for the high-risk group.

$P<0.001)$ with a relatively good accuracy $(\mathrm{C}=0.63)$. Median survival time was significantly shorter $(P=0.001)$ in the high-risk group (1.11 years) when compared to the low-risk group (2.26 years). Figure 1 represents the Kaplan-Meier survival curves for the high- and low-risk groups in the discovery sample, and reveals a statistically significant increase in mortality among patients in the high-risk category.

\section{Replication Analyses}

Cox analyses revealed that the risk score replicated well $(\mathrm{HR}=8.39,[2.61-26.97], P<0.001)$ with relatively good model fit $(\mathrm{C}=0.65)$. Stratifying into high- and low-risk groups showed similar shortened survival in the high-risk group (Figure 2). Cox proportional hazards analyses also suggested that the high-risk group had reduced survival

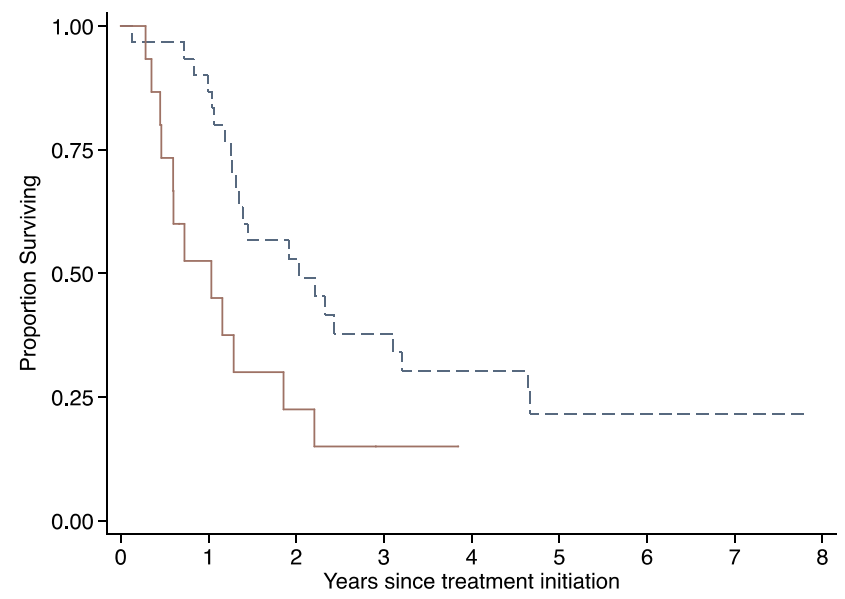

Figure 2 Kaplan-Meier analysis of survival risk in the high- and low-risk groups in the replication sample. Dashed line shows survival for the low-risk group, while solid line shows survival for the high-risk group. 
(HR=2.40, [1.16-4.96], $P=0.019)$ with similar overall ability $(\mathrm{C}=0.62)$. Median survival time was shorter $(P=0.035)$ in the high-risk group ( 0.72 years) when compared to the low-risk group (1.86 years).

Sensitivity analyses carried out in the discovery sample revealed that the risk score worked well when limiting the sample to patients enrolled at stage III $(\mathrm{HR}=3.54$, [1.48-8.43], $P=0.004$ ) and also if histology was adenocarcinoma $(\mathrm{HR}=4.08,[1.31-12.67], P=0.015)$ or squamous-cell carcinoma $(\mathrm{HR}=9.94,1.64-60.25], P=0.012)$.

\section{Discussion}

Prognostic factors provide valuable information about disease evolution and may sometimes be useful for guiding therapy. The presence of poor prognostic factors could help identify subgroups of patients who might benefit from more aggressive therapy, and may also provide patients a better understanding of their own prognosis before making treatment decisions. Although there are some well-established prognostic factors for advanced NSCLC, there have been conflicting results from studies in stage III NSCLC patients treated with chemoradiotherapy. Our analysis used novel methods to demonstrate that demographic factors (age, sex), clinical factors (albumin levels, histology, stage), and treatment factors (radiation dose, induction therapy) all played a role in determining survival. Additionally, we were able to reliably predict survival based on our prediction model and classify patients into high- and low-risk groups. Median survival in the low-risk group was more than two years, compared to less than one year in the high-risk group. To our knowledge, this is the first study to describe a risk stratification model to predict survival in patients with locally advanced NSCLC treated with chemoradiotherapy. Clinicians can use this model to devise a personalized treatment plan for their patients, possibly avoid additional therapy in the low-risk group, and implement strategies for risk reduction in the high-risk group.

Jeremic and Shibamoto evaluated prognostic factors among 169 stage III NSCLC patients treated with hyperfractionated thoracic radiotherapy TRT (64-68 Gy) with or without chemotherapy. In that study, Karnofsky PS $\geq 80 \%$, weight loss $\leq 5 \%$, lower disease stage, younger age $(<60$ years), and female gender were all associated with an improved survival. ${ }^{12}$ Socinski et al analyzed the Cancer and Leukemia Group B (CALGB) database to evaluate the influence of various factors on survival and toxicity. In their analysis, baseline hemoglobin, PS, and the use of combined modality therapy had the greatest impact on survival in unresectable stage III NSCLC. ${ }^{13}$

FDG-PET has significantly improved the accuracy of detection of involved lymph nodes and distant metastases. Kramer et al demonstrated that PET-based staging was a better predictor of survival compared to non-PET-based TNM staging. ${ }^{14}$ In a European study, the investigators evaluated the prognostic value of tumor volume and $\mathrm{N}$ status in NSCLC patients treated with chemoradiation. In this retrospective analysis of 270 patients with inoperable NSCLC, the number of positive lymph node stations, as well as the $\mathrm{N}$ stage on PET scan, was associated significantly with survival. The median survival for patients with no positive lymph node stations (PLNS) was 20 months, compared to 11 months for those with 3 or more PLNS $(P<0.001) .{ }^{12}$ The nodal station information in our study was extracted from pre-treatment FDG-PET.

Our data are consistent with prior studies with regards to stage and number of nodal stations, but we did not find PS to be predictive of survival. This could be due to the fact there were only a small number of patients with poor PS in our study; due to the small sample size, a statistically significant difference could not be observed $(\mathrm{HR}=1.21$, [95\% (CI) 0.83-1.73]; $P<0.32$ ).

While adenocarcinoma is the most commonly encountered histology, in our study there were approximately $40 \%$ of patients with squamous cell carcinoma, $40 \%$ with adenocarcinoma, and $20 \%$ with otherwise unspecified histology. Histology may be an independent prognostic factor, but results have been equivocal from various studies. ${ }^{15,16}$ In our investigation, histology was identified as an important prognostic factor.

Retrospective studies have suggested that higher radiation dose is associated with improved local control and survival rates. ${ }^{17,18}$ A dose of $60-63 \mathrm{~Gy}$ in 1.8 to $2.0 \mathrm{~Gy}$ fraction sizes is the commonly accepted standard radiation dose based on the RTOG 7301 trial. ${ }^{19}$ Our findings were in accordance with published literature; patients who received a radiation dose greater than or equal to $60 \mathrm{~Gy}$ had better survival than those who received less than 60 Gy. The subsequent RTOG 0617 trial showed that 60 Gy was superior to $74 \mathrm{~Gy}$ in OS and loco-regional control. ${ }^{20}$

Prior studies have failed to demonstrate the survival advantage of either induction or consolidation chemotherapy in this patient population. ${ }^{4,5}$ Our study revealed that people who received induction chemotherapy had better survival and were categorized in the low-risk group based on the model. However, this finding is somewhat limited 
by the small number of patients who received induction chemotherapy.

Weight loss has also been frequently evaluated has an important prognostic factor. ${ }^{17,21}$ In a systematic review of the predictive value of pre-treatment serum albumin in multiple cancer types, Gupta and Lis demonstrated that serum albumin has a strong prognostic role in predicting cancer survival. ${ }^{22}$ The present study confirmed this finding.

\section{Limitations}

Although our study used novel methods, there are some limitations. First, this is a relatively small study that lacked external replication data. However, this sample represents all the patients with locally advanced NSCLC treated with CRT at our institution between 2007 and 2015. While we made every effort to improve replicability by utilizing an internal replication sample, future studies are needed to validate these findings in external samples and also in patients getting immunotherapy as consolidation. Second, although the study was of sufficient power to enable these analyses in part because many of those who were treated died in a relatively short period of time, a larger sample may be useful in improving predictive reliability of uncommon risk factors. Third, this study has the inherent bias of a retrospective study design, including uncertainties due to missing data.

Fourth, even though the total radiation dose of 60-66 Gy was planned for all patients, most patients received a total radiation dose $\leq 60 \mathrm{~Gy}$. The exact reason in not clear but early treatment discontinuation due to poor tolerance, missing data, and transfer of care to another facility can account for this discrepancy.

Lastly, the study population was heterogeneous, as it included medically inoperable stage II patients, patients with mediastinal recurrence after resection of the primary tumor, and oligo-metastatic stage IV patients who received definitive therapy for the single metastatic site. Given these limitations, it is unclear how well these results would apply to patients presenting de novo with stage III NSCLC.

\section{Future Directions}

Although our model is robust and accurate, these findings need to be validated in the cohort getting consolidation immunotherapy. Furthermore, this model could potentially be applied to determine subsets of patients who may benefit less from durvalumab, or for whom more aggressive consolidative treatment might be more appropriate. Molecular markers should also be incorporated into the model and alternative consolidation regimens should be evaluated for high-risk patients.

Further studies should also aim at refining the radiation techniques, treatment planning systems, and possibly boost RT to bulky disease areas.

\section{Conclusion}

The current study is one of the first to report a risk stratification model based on prognostic factors implicated in the progression of locally advanced NSCLC patients treated with chemo-radiation. This unique model can help oncologists devise a personalized treatment plan for these patients. Future studies are needed to validate these findings prospectively on a larger scale, utilizing external data, and in patients getting consolidation immunotherapy. The current model can be further improved by incorporating genomics, proteomics, and radiomics analysis to explore new prognostic factors. ${ }^{23-26}$

\section{Data Sharing Statement}

All data generated or analyzed during this study are included in this published article.

\section{Ethics Approval}

The study was approved by the institution's Committee on Research Involving Human Subjects (CORIHS). Stony Brook IRB waived the consent due to the retrospective nature of the study. All data were compiled in a Microsoft Excel spreadsheet, which was password protected to maintain patient confidentiality. Each subject was assigned a unique number in consecutive order, not derived from any patient identifiers. There was no disclosure of the Personal Health Information (PHI) and study was conducted in compliance with the Declaration of Helsinki.

\section{Author Contributions}

All authors made substantial contributions to conception and design, acquisition of data, or analysis and interpretation of data; took part in drafting the article or revising it critically for important intellectual content; gave final approval of the version to be published; and agree to be accountable for all aspects of the work.

\section{Disclosure}

The authors report no funding and no conflicts of interest for this work. 


\section{References}

1. Siegel RL, Miller KD, Jemal A. Cancer statistics, 2019. CA Cancer J Clin. 2019;69(1):7-34. doi:10.3322/caac.21551

2. Dillman RO, Herndon J, Seagren SL, et al. Improved survival in stage III non-small-cell lung cancer: seven-year follow-up of cancer and leukemia group B (CALGB) 8433 trial. J Natl Cancer Inst. 1996;88(17):1210-1215. doi:10.1093/jnci/88.17.1210

3. Gandara DR, Chansky K, Albain KS, et al. Consolidation docetaxel after concurrent chemoradiotherapy in stage IIIB non-small-cell lung cancer: phase II Southwest Oncology Group Study S9504. J Clin Oncol. 2003;21(10):2004-2010. doi:10.1200/JCO.2003.04.197

4. Hanna N, Neubauer M, Yiannoutsos C, et al. Phase III study of cisplatin, etoposide, and concurrent chest radiation with or without consolidation docetaxel in patients with inoperable stage III nonsmall-cell lung cancer: the Hoosier Oncology Group and U.S. Oncology. J Clin Oncol. 2008;26(35):5755-5760. doi:10.1200/ JCO.2008.17.7840

5. Vokes EE, Herndon JE, Kelley MJ, et al. Induction chemotherapy followed by chemoradiotherapy compared with chemoradiotherapy alone for regionally advanced unresectable stage III non-small-cell lung cancer: cancer and leukemia Group B. J Clin Oncol. 2007;25 (13):1698-1704. doi:10.1200/JCO.2006.07.3569

6. Belani CP, Choy H, Bonomi P, et al. Combined chemoradiotherapy regimens of paclitaxel and carboplatin for locally advanced nonsmall-cell lung cancer: a randomized phase II locally advanced multimodality protocol. $J$ Clin Oncol. 2005;23(25):5883-91. 22. doi:10.1200/JCO.2005.55.405

7. Antonia SJ, Villegas A, Daniel D, et al. Durvalumab after chemoradiotherapy in stage III non-small-cell lung cancer. $N$ Engl J Med. 2017;377(20):1919-1929. doi:10.1056/NEJMoa1709937

8. Firat S, Byhardt RW, Gore E, Radiation Therapy Oncology Group. Comorbidity and karnofksy performance score are independent prognostic factors in stage III non-small-cell lung cancer: an institutional analysis of patients treated on four RTOG studies. Int J Radiat Oncol Biol Phys. 2002;54(2):357-364. doi:10.1016/S0360-3016(02)02939-5

9. Ademuyiwa FO, Johnson CS, White AS, et al. Prognostic factors in stage III non-small-cell lung cancer. Clin Lung Cancer. 2007;8 (8):478-482. doi:10.3816/CLC.2007.n.031

10. Zou H, Hastie T. Regularization and variable selection via the elastic net. J R Stat Soc Series B Stat Methodol. 2005;67(2):301-320. doi:10.1111/j.1467-9868.2005.00503.x

11. Youden WJ. Index for rating diagnostic tests. Cancer. 1950;3 (1):32-35. doi:10.1002/1097-0142(1950)3:1<32::AIDCNCR2820030106 $>3.0 . \mathrm{CO} ; 2-3$

12. Jeremic B, Shibamoto Y. Pre-treatment prognostic factors in patients with stage III non-small cell lung cancer treated with hyperfractionated radiation therapy with or without concurrent chemotherapy. Lung Cancer. 1995;13(1):21-30. doi:10.1016/0169-5002(95)00480-O

13. Socinski MA, Zhang C, Herndon JE, et al. Combined modality trials of the Cancer and Leukemia Group B in stage III non-small-cell lung cancer: analysis of factors influencing survival and toxicity. Ann Oncol. 2004;15(7):1033-1041. doi:10.1093/annonc/mdh282
14. Kramer H, Post WJ, Pruim J, et al. The prognostic value of positron emission tomography in non-small cell lung cancer: analysis of 266 cases. Lung Cancer. 2006;52(2):213-217. doi:10.1016/j.lungcan.2005.12.011

15. Werner-Wasik M, Scott C, Cox JD, et al. Recursive partitioning analysis of 1999 radiation therapy oncology group (RTOG) patients with locally-advanced non-small-cell lung cancer (LA-NSCLC): identification of five groups with different survival. Int $J$ Radiat Oncol Biol Phys. 2000;48(5):1475-1482. doi:10.1016/S03603016(00)00801-4

16. Basaki K, Abe Y, Aoki M, et al. Prognostic factors for survival in stage III non-small-cell lung cancer treated with definitive radiation therapy: impact of tumor volume. Int J Radiat Oncol Biol Phys. 2006;64(2):449-454. doi:10.1016/j.ijrobp.2005.07.967

17. Brundage MD, Davies D, Mackillop WJ. Prognostic factors in non-small cell lung cancer: a decade of progress. Chest. 2002;122 (3):1037-1057. doi:10.1378/chest.122.3.1037

18. Solan MJ, Werner-Wasik M. Prognostic factors in non-small cell lung cancer. Semin Surg Oncol. 2004;15(7):64-73. doi:10.1002/ssu.10023

19. Perez CA, Stanley K, Rubin P, et al.. A prospective randomized study of various irradiation doses and fractionation schedules in the treatment of inoperable non-oat-cell carcinoma of the lung. Preliminary report by the radiation therapy oncology group. Cancer. 1980;45 (11):2744-2753. doi:10.1002/1097-0142(19800601)45:11<2744:: AID-CNCR2820451108>3.0.CO;2-U

20. Bradley JD, Paulus R, Komaki R, et al. Standard-dose versus high-dose conformal radiotherapy with concurrent and consolidation carboplatin plus paclitaxel with or without cetuximab for patients with stage IIIA or IIIB non-small-cell lung cancer (RTOG 0617): a randomised, two-by-two factorial Phase 3 study. Lancet Oncol. 2015;16(2):187-199. doi:10.1016/S1470-2045(14)71207-0

21. Mantzorou M, Koutelidakis A, Theocharis S, et al. Clinical value of nutritional status in cancer: what is its impact and how it affects disease progression and prognosis? Nutr Cancer. 2017;69 (8):1151-1176. doi:10.1080/01635581.2017.1367947

22. Gupta D, Lis CG. Pretreatment serum albumin as a predictor of cancer survival: a systematic review of the epidemiological literature. Nutr J. 2010;9(1):69. doi:10.1186/1475-2891-9-69

23. Lambin P, Rios-Velazquez E, Leijenaar R, et al. Radiomics: extracting more information from medical images using advanced feature analysis. Eur J Cancer. 2012;48(4):441-446. doi:10.1016/j.ejca.2011.11.036

24. Langendijk H, Thunnissen E, Arends JW, et al. Cell proliferation and apoptosis in stage III inoperable non-small cell lung carcinoma treated by radiotherapy. Radiother Oncol. 2000;56(2):197-207. doi:10. 1016/S0167-8140(00)00218-8

25. Mascaux C, Iannino N, Martin B, et al. The role of RAS oncogene in survival of patients with lung cancer: a systematic review of the literature with meta-analysis. $\mathrm{Br} J$ Cancer. 2005;92(1):131-139. doi:10.1038/sj.bjc.6602258

26. Berghmans T, Meert AP, Martin B, Ninane V, Sculier JP. Prognostic role of epidermal growth factor receptor in stage III nonsmall cell lung cancer. Eur Respir J. 2005;25(2):329-335. doi:10.1183/090319 36.05.00060804
Cancer Management and Research

\section{Publish your work in this journal}

Cancer Management and Research is an international, peer-reviewed open access journal focusing on cancer research and the optimal use of preventative and integrated treatment interventions to achieve improved outcomes, enhanced survival and quality of life for the cancer patient.
The manuscript management system is completely online and includes a very quick and fair peer-review system, which is all easy to use. Visit http://www.dovepress.com/testimonials.php to read real quotes from published authors. 